\title{
Cervical spontaneous hematoma in elderly woman:
}

\section{case report}

\begin{abstract}
Background: Spontaneous cervical hematomas are rare entities. In the absence of previous trauma they have been described in patients with blood dyscrasias or in treatment with anticoagulants or antiaggregants.
\end{abstract}

Case report: We present a case of spontaneous cervical hematoma without respiratory compromise in a 97-year-old woman who was treated in a non-operative manner in our center in a satisfactory way.

Discussion: The main risk of cervical hematoma is the compression of the airway with the risk of asphyxia that it can generate. In patients hemodynamically stable and without respiratory compromise, conservative management can be performed. However, patients with significant anemia and / or respiratory compromise require correct airway management (orotracheal intubation), drainage of the hematoma and hemostasis of the bleeding vessel.

Keywords: cervical hematomas, orotracheal intubation, valsalva maneuvers, multinodular goiter, hemodynamically, anticoagulants, antiaggregants, coughing, sneezing, defecation, ipsilateral laterocervical, bronchopulmonary dysplasia, erythema, petechiae
Volume 6 Issue 5 - 2018

\author{
Mendoza-Moreno Fernando,' Furtado-Lobo \\ Isabel,' Matías-García Belen,' Díez-Gago \\ María del Rocío, ${ }^{2}$ Blázquez-Martín Alma,' \\ Díez-Alonso Manuel,' Hernández Merlo \\ Francisco,' Noguerales-Fraguas Fernando' \\ 'Department of General and Digestive Surgery, Príncipe de \\ Asturias Teaching Hospital, Alcalá de Henares, Spain \\ ${ }^{2}$ Department of Emergency Medicine, Príncipe de Asturias \\ Teaching Hospital,Alcalá de Henares, Spain
}

Correspondence: Fernando Mendoza-Moreno, Príncipe de Asturias Teaching Hospital, Alcalá de Henares, Madrid, Spain, Tel +00349|8878। 00, Email khoril@hotmail.com

Received: June 29, 2018 | Published: September 18, 2018

\section{Introduction}

Spontaneous cervical hematomas are rare entities. Although the thyroid gland is a highly vascular organ, few cases have been described in the literature. ${ }^{1}$ More frequent are hematomas of traumatic origin, such as those that originate on mediastinal vessels or those related to the insertion of venous catheters at the jugular level. ${ }^{2}$ Among the causes of spontaneous cervical hematoma are the enlargement of the thyroid gland or the appearance of thyroid nodules as in the case of multinodular goiter (the increase in the size of the gland is linked to an increase in its vascularization), hemodynamic alterations (such as treatment with dialysis) or coagulation (use of heparin, anticoagulant or antiaggregant drugs) or intrathoracic pressure increase such as that produced during Valsalva maneuvers (coughing, sneezing, defecation etc). ${ }^{3}$

Here we report a case of spontaneous cervical hematoma without respiratory symptoms or previous documented trauma, resolved by non-operative treatment in a 97-year-old woman.

\section{Case report}

A 97-year-old woman was admitted to hospital with increased volume at the cervical level without associated respiratory symptoms. She did not report a history of previous trauma on the area. Her medical history was significant for hypertension and atrial fibrillation in treatment with bisprolol ( $2.5 \mathrm{mg} / 24$ hours) and acetylsalicylic acid (100 mg / 24 hours) respectively. Vital signs were 107/47 mm Hg for blood pressure, $58 \mathrm{bpm}$ and oxygen saturation of $93 \%$. Physical examination showed a hematoma in the cervical region without respiratory compromise of the airway (Figure 1).

The blood tests showed an INR of 1.09 (normal laboratory value $0.90-1.22)$ and a hemoglobin of $11.2 \mathrm{gr} / \mathrm{dl}(12 \mathrm{gr} / \mathrm{dl}-16 \mathrm{gr} / \mathrm{dl})$ with the rest of the laboratory parameters normals. A chest x-ray was performed that no reveal significant findings (Figure 2).
Inmmediately, a cervical CT scan was made that showed a parenchymal laceration of the right thyroid lobe with hematoma evolved in the ipsilateral laterocervical region and in the superior mediastinum without evidence of compatible images with aneurysms in the arterial territories studied (Figure 3).

As the patient was stable at the moment of diagnosis it was decided to admit to the hospital for monitoring and watching. The following day, a control blood tests were made, in where the decrease of one hemoglobin point was observed $(10.3 \mathrm{gr} / \mathrm{dl})$, so transfusion of blood was not necessary. The patient was in digestive rest, with fluid therapy for the first 3 days. From the fourth day and given the respiratory and hemodynamic stability, it was decided to start the oral intake with good tolerance. During her stay in the hospital, the patient remained hemodynamically stable without respiratory symptoms, being discharged after 72 hours of hospitalization.

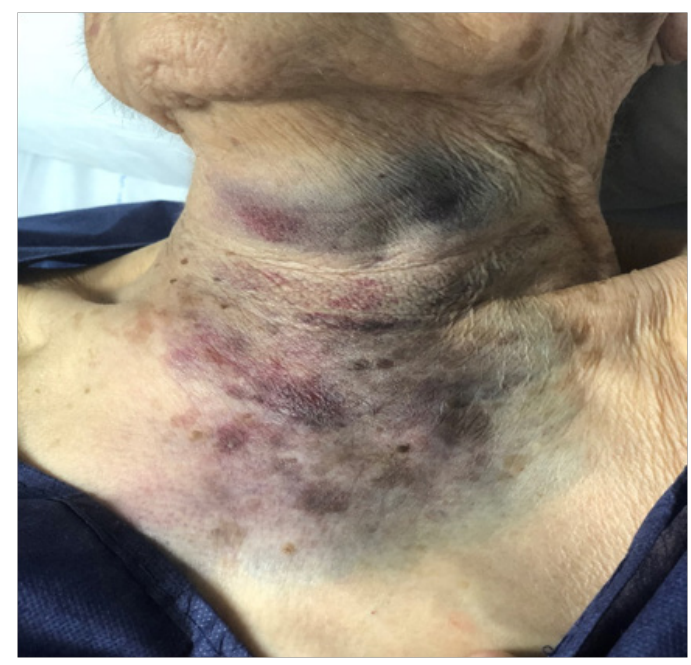

Figure I Spontaneous cervical hematoma in elderly women. 


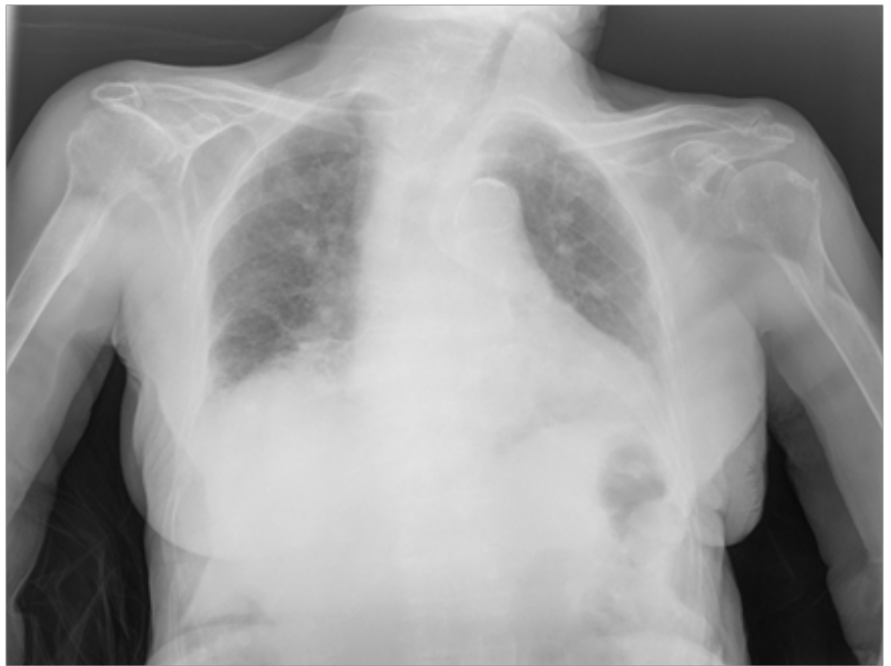

Figure 2 Normal chest X-Ray

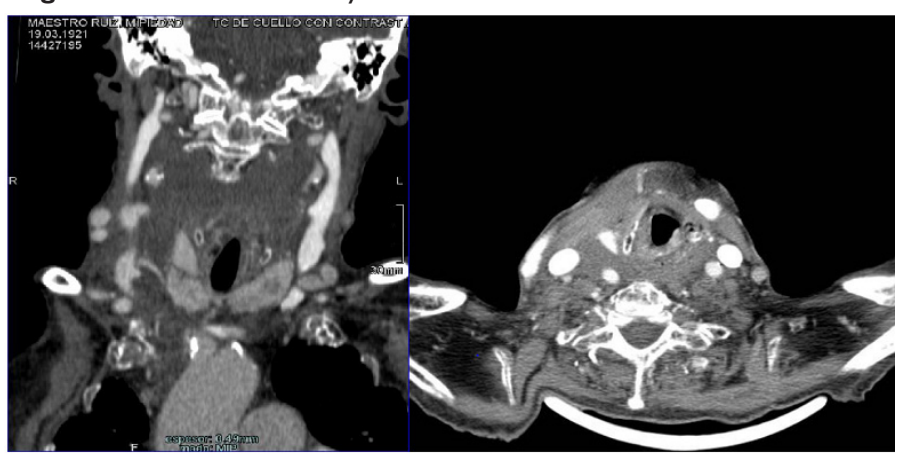

Figure 3 CT scan that shows cervical hematoma and thyroid disruption.

\section{Discussion}

Cervical spontaneous hematoma is a rare condition. With a documented traumatic mechanism, mediastinal hematomas usually originate after thoracic trauma or rupture of the aneurysmal aorta while the cervical ones (with normal thyroid gland) have been described after vertebral body fractures, thyroid surgery, fine needle aspiration, deceleration sudden or cervical hyperflexion. ${ }^{3-6}$

In addition to the aforementioned causes of spontaneous hematoma, it has been described in patients with multinodular goiter, since the presence of thyroid nodules is accompanied by an increase in thyroid vascularization. Some authors such as Boris have described this entity in the immediate postpartum. ${ }^{7}$ Coskun, described them after rupture of an aneurysm. ${ }^{8}$ Aneurysms of branches of supra-aortic trunks are extremely rare. However, the inferior thyroid artery is the arterial vessel on which a greater number of aneurysms have been described. ${ }^{8}$ Hanashiro, describes the rupture of the inferior thyroid artery without the presence of aneurysm, spontaneously originating a hematoma in the neck and mediastinum. ${ }^{9}$ In relation to the cases described in anticoagulated patients, only one third of them had overdose of anticoagulant drug levels. However, the most frequent cause of spontaneous cervical hematoma described in the literature is persistent cough. ${ }^{6}$ A massive hemorrhage on the thyroid cell can expand to the parapharyngeal or retropharyngeal space causing tracheal compression and therefore compromise of the airway originating a true surgical emergency. However, a minority of patients may be asymptomatic and managed conservatively as the case described in our experience. ${ }^{10}$

For diagnosis, clinical suspicion of increased cervical volume and respiratory work is enough. A contrast-enhanced CT scan is the preferred option for diagnosis in stable patients without respiratory compromise, as it is a fast and non-invasive method that allows an adequate evaluation of the cervical region, the mediastinum, its main arterial vessels and its branches. ${ }^{6}$ The performance of an angiography can be useful because, as a diagnostic method, it also allows embolization of the bleeding vessel. A cervical hematoma is a potential cause of death. At mediastinal level it can cause a cardiac tamponade or at the cervical level compress the airway until its collapse. ${ }^{2}$ The compression of the airway is a surgical emergency, causing respiratory distress, cervical or thoracic ecchymosis, hypotension, tachycardia or dyspnea. In symptomatic patients, the first step should be to control the airway, usually by orotracheal intubation or as described in some cases, by tracheostomy. Next, an exploratory cervicotomy is performed to drain the hematoma and control the hemostasis. In some cases, before a large hematoma originating in the thyroid gland, may require hemithyroidectomy or even total thyroidectomy. In asymptomatic patients, non-operative management consisting of surveillance, monitoring, antibiotherapy (for immunosuppressed patients) and use of steroids has been described. ${ }^{2,9}$

In conclusion, spontaneous cervical hematomas are infrequent entities that can constitute a true surgical emergency, requiring generally in addition to control of the airway, surgical treatment in most cases.

\section{Acknowledgements}

None.

\section{Conflict of interest}

All authors declare no conflict of interest.

\section{References}

1. Gunasekaran K, Rudd KM, Murthi S, et al. Spontaneous Thyroid Hemorrhage on Chronic Anticoagulation Therapy. Clin Pract. 2017;7(1):932.

2. Hoetzenecker K, Topker M, Klepetko W, et al. Spontaneous rupture of the inferior thyroid artery resulting in mediastinal hematoma. Interact Cardiovasc Thorac Surg. 2010;11(2):209-210.

3. Amadei EM, Benedettini L, Piccin O. Two cases of cervical hemorrhage with upper airway obstruction: a life-threatening condition. Case Rep Med. 2014: Article ID 674176

4. Sahin S, Belice T, Ogullar S, et al. Syncope in a patient with spontaneous hemorrhage into a thyroid nodule. Hippokratia. 2014;18(2):177-179.

5. Mikubo M, Sonoda D, Yamazaki H, et al. Spontaneous non-traumatic mediastinal hematoma associated with oral anticoagulant therapy: A case report and literature review. Int J Surg Case Rep. 2017;39:221-224.

6. Li X, Liu L, Cao D, et al. Spontaneous Hematoma of Posterior Mediastinum with an Uncommon Cause: A Case Report and Review of the Literature. Clin Pract. 2016;6(1):838.

7. Hristov BD, Borrego R, Harding PA, et al. A Case of Spontaneous Postpartum Thyroid Hemorrhage Leading to Upper Airway Obstruction. Am J Case Rep. 2016;17:192-195.

8. Coskun ZO, Yavaşi Ö, Durakoglugil T, et al. Acute airway compromise due to ruptured inferior thyroid artery aneurysm. Am J Emerg Med. 2015;33(8):1115.e1-3. 
9. Hanashiro N, Yamashiro T, Iraha Y, et al. Non-traumatic rupture of the superior thyroid artery with concomitant parathyroid adenoma and multinodular goiter. Acta Radiol Open. 2017;6(7):1-4.
10. Chida Y, Inokuchi R, Ishida T, et al. Spontaneous mediastinal haematoma. BMJ Case Rep. 2016; bcr2016217799. 